\title{
Risk Aversion in Cumulative Prospect Theory
}

\author{
Ulrich Schmidt ${ }^{\mathrm{a}} \&$ Horst Zank ${ }^{\mathrm{b}, *}$ \\ anstitut für Finanzwissenschaft und Sozialpolitik, Christian-Albrechts-Universität zu \\ Kiel, Germany. \\ ${ }^{b}$ School of Economic Studies, The University of Manchester, United Kingdom.
}

20 November, 2001

Proposed Running Title: Risk Aversion in CPT

${ }^{*}$ Corresponding author: Horst Zank, School of Economic Studies, The University of Manchester, Oxford Road, Manchester M13 9PL, United Kingdom; Telephone: ++44 161275 4872, Fax: ++44 161 275 4812, E-mail: horst.zank@man.ac.uk 
Abstract. This paper characterizes the conditions for risk aversion in cumulative prospect theory where risk aversion is defined in the strong sense $([27])$. Under weaker assumptions than differentiability we show that risk aversion implies convex weighting functions for gains and for losses but not necessarily a concave utility function. Also, we investigate the exact relationship between loss aversion and risk aversion. We illustrate the analysis by considering two special cases of cumulative prospect theory and show that risk aversion and convex utility may coexist.

Keywords: cumulative prospect theory, strong risk aversion, loss aversion, convex utility. Journal of Economic Literature Classification Number D81 


\section{Introduction}

Cumulative prospect theory (CPT) has nowadays become the most prominent alternative to expected utility (EU). It is widely used in empirical research and various axiomatic characterizations of CPT have been proposed ( [21], [22], [33], [34], [5], [29], and [30]). The paradoxes of Allais [1] and Ellsberg [11] are resolved under CPT, as well as the coexistence of gambling and insurance ([13]). The equity premium puzzle ([23]), the overtime premium puzzle $([8])$, the status quo bias $([28])$, and the endowment effect $([32])$ can all be accommodated under CPT.

Another popular alternative to expected utility is the rank-dependent utility (RDU) model of Quiggin [25]. RDU generalizes EU by introducing a weighting function which transforms cumulative probabilities. CPT is even more general than RDU by allowing additionally for sign-dependence (there exist two separate weighting functions, one for gains and one for losses, which do not need to coincide) and reference-dependence (utility is defined on deviations from a status quo, i.e. on gains and losses, and not on final wealth positions). Due to reference-dependence a decision maker in the CPT framework can exhibit loss aversion which means that the utility of a loss weights more heavily than the utility of a corresponding gain.

There exist various theoretical applications of RDU in the literature, especially in the context of insurance economics. These applications have shown that RDU generates results which differ substantially from those derived in the expected utility framework and in many cases these results provide a better accommodation of observed data. In general, the theoretical applications of RDU - and even those of EU - assume strong risk aversion. An individual exhibits strong risk aversion if she or he always dislikes mean- 
preserving spreads in risk (cf. [27]). Chew, Karni and Safra [6] have shown that strong risk aversion is satisfied within the RDU framework if and only if the utility function is concave and the weighting function is convex. In contrast, strong risk aversion has not yet been analyzed for CPT and the goal of the present paper is to fill this gap. It is useful to derive the conditions for strong risk aversion under CPT in order to improve our understanding of this model. Moreover, since most theoretical applications of RDU and EU assume strong risk aversion it is reasonable to do the same for CPT in order to make the results comparable.

Our main result shows that the conditions for strong risk aversion under CPT and RDU coincide if we consider only gains or if we consider only losses, i.e. utility is concave on the domain of gains and also concave in the domain of losses and both weighting functions are convex. However, this result generalizes the finding of Chew, Karni, and Safra [6] since we do not employ any differentiability assumption. If gains and losses are considered simultaneously, surprisingly utility does not need to be concave; in extreme cases strong risk aversion and convex utility may coexist under CPT. This result is noteworthy since Chateauneuf and Cohen [3] tried to derive the coexistence of risk aversion and convex utility under RDU. They have shown that RDU is compatible with convex utility only in the presence of weak risk aversion (i.e. any lottery is dispreferred to its expected value) while strong risk aversion forces utility to be concave everywhere. Moreover, our results show that there exists a particular relationship between strong risk aversion and loss aversion: in general, strong risk aversion is compatible with loss seeking, while utility is concave if and only if loss aversion holds. Surprisingly, the relationship between strong risk aversion and loss aversion is characterized by the ratio of the left and right derivative of the utility function at zero. Theoretical arguments have motivated Köbberling and 
Wakker [18] to propose this ratio as index of loss aversion. Our results support their proposal since in our framework this index of loss aversion arises naturally.

In order to shed some more light on the consequences of strong risk aversion in the CPT framework we consider two specific variants of the model. In the first variant utility is a linear/exponential function, and in the second case it is a power function. By incorporating strong risk aversion these variants lead to examples where the utility function is non-concave, in particular loss seeking behavior is allowed in a region around the status quo. In the case of the power function utility becomes linear for gains and linear for losses with a possible kink at the status quo.

One may argue that the analysis of loss aversion in the CPT framework is of less interest since the value function in prospect theory and CPT is usually proposed to be convex in order to accommodate empirical observed risk seeking for losses ([16], [33]). However, many empirical studies found linear utility for small losses ([10], [15], [31], [7], [36], and [20]). Moreover, it seems to be commonly agreed that utility is linear, at least for small stakes ([19], [12], [17]). Theoretical arguments for linear utility have been provided by Hansson [14] and Rabin [26]. Additionally, there seems to be some evidence that a decision maker, who initially is risk seeking in the loss domain, changes attitudes towards risk while gaining experience. This fact has lead Myagkov and Plott [24] to formulate the conjecture that "with experience, risk seeking in the losses evolves into either risk-neutral or risk-averse behavior." These empirical findings as well as the fact that the theoretical applications of RDU and EU assume strong risk aversion indicate that strong risk aversion should also be analyzed in the CPT framework.

The paper is organized as follows. In the next section we present the CPT model and derive our main results. Section 3 considers the consequences of strong risk aversion 
for the two special variants of CPT mentioned above. All proofs are presented in the Appendix.

\section{Cumulative Prospect Theory and Risk Aversion}

In this section we recall the general cumulative prospect theory model for decision under risk. It is assumed that a decision maker has a preference relation over lotteries. A lottery is a finite probability distribution over the set of monetary outcomes (here identified with the set of real numbers, $\mathbb{R})$. It is represented by $P:=\left(p_{1}, x_{1} ; \ldots ; p_{n}, x_{n}\right)$ meaning that probability $p_{j}$ is assigned to outcome $x_{j}$, for $j=1, \ldots, n$. The probabilities $p_{j}$ are nonnegative and sum to one. With this notation we implicitly assume that outcomes are ranked in decreasing order, i.e., $x_{1} \geqslant \cdots \geqslant x_{n}$. Without loss of generality, we assume that the status quo is given by zero. Therefore, we refer to positive outcomes as gains and to negative outcomes as losses.

Cumulative Prospect Theory (CPT) holds if the decision maker evaluates lotteries by the following functional.

$$
\left(p_{1}, x_{1} ; \ldots ; p_{n}, x_{n}\right) \mapsto \sum_{j=1}^{n} \pi_{j} U\left(x_{j}\right)
$$

where $U$ is the utility function and the $\pi_{j}$ 's are decision weights.

The utility function assigns to each outcome a real value, in particular $U(0)=0$, and it is assumed that utility is strictly increasing and continuous.

The decision weights are generated by probability weighting functions $w^{+}, w^{-}$. These functions map the interval of probabilities $[0,1]$ into itself, they are strictly increasing and satisfy $w^{+}(0)=w^{-}(0)=0$, and $w^{+}(1)=w^{-}(1)=1$. For a lottery $\left(p_{1}, x_{1} ; \ldots ; p_{n}, x_{n}\right)$, the decision weights are defined as follows. There exists some $k \in\{0, \ldots, n\}$ such that 
$x_{1} \geqslant \cdots \geqslant x_{k} \geqslant 0>x_{k+1} \geqslant \cdots \geqslant x_{n}$. Then

$$
\pi_{j}= \begin{cases}w^{+}\left(p_{1}+\cdots+p_{j}\right)-w^{+}\left(p_{1}+\cdots+p_{j-1}\right), & \text { if } j \leqslant k, \\ w^{-}\left(p_{1}+\cdots+p_{j}\right)-w^{-}\left(p_{1}+\cdots+p_{j-1}\right), & \text { if } j>k .\end{cases}
$$

Under CPT utility is a ratio scale, i.e., it is unique up to multiplication by a positive constant, and the weighting functions are uniquely determined.

Several axiomatizations of CPT can be found in the literature. To derive the general functional form often complex conditions are required beyond the standard properties (continuity, weak ordering, stochastic dominance). Luce and Fishburn [22] use a condition termed compound gamble and joint receipt (see also [21]). Tversky and Kahneman [33], Wakker and Tversky [34], Chateauneuf and Wakker [5] and Schmidt [29] use signdependent comonotonic tradeoff-consistency. The conditions can be less complex if a particular parametric form for utility is desired. Wakker and Zank [35] use a generalization of constant proportional risk aversion to incorporate losses and derive CPT with utility as power function. Zank [37] provides a model where utility is exponential or linear by requiring constant absolute risk aversion for gains and separately for losses. These two models are analyzed in more detail in the next section. Schmidt and Zank [30] use a condition called independence of common increments to derive a model where utility is linear for losses and linear for gains.

Under expected utility risk aversion is equivalent to requiring concavity of the utility function. Since the flow of alternatives to expected utility theory many alternative notions of risk aversion have been proposed and analyzed. The most prominent one defines risk aversion as aversion to mean preserving spreads which is referred to as strong risk aversion. This concept of risk aversion was introduced by Rothschild and Stiglitz [27]. More precisely, an individual exhibits strong risk aversion if for all lotteries $P=\left(p_{1}, x_{1} ; \ldots ; p_{n}, x_{n}\right)$ 
and all $\delta>0$ it follows that

$$
\left(p_{1}, x_{1} ; \ldots ; p_{i}, x_{i}-\frac{\delta}{p_{i}} ; \ldots ; p_{j}, x_{j}+\frac{\delta}{p_{j}} ; \ldots ; p_{n}, x_{n}\right) \succcurlyeq P
$$

whenever $p_{i}, p_{j}>0$. Recall that due our notation $\delta$ must be chosen such that rankordering of outcomes is maintained.

Several generalizations of strong risk aversion have been proposed by Chateauneuf, Cohen, and Meilijson [4], and their implications on various decision models have been studied. Strong risk aversion is a property which is model independent - i.e. defined in terms of preferences and not in terms of properties of the utility representation - and this fact may explain its popularity. In the next theorem we present the implications of strong risk aversion under general CPT. Some of our results are similar to those found for RDU, which is the special case of CPT given by $w^{+} \equiv w^{-}$. As shown by Chew, Karni, and Safra [6], in the case of RDU utility has to be concave and the weighting function has to be convex in order to satisfy strong risk aversion. In the theorem below we do not assume any differentiability of the utility function, nor do we assume continuity of the weighting functions on $[0,1]$. We rather adopt the approach of Ebert [9], who has shown in a welfare theory setting that these assumptions can be relaxed. In this sense Theorem 1 below also generalizes the existing results for rank-dependent expected utility since Chew, Karni, and Safra [6] assumed Gâteaux-differentiability.

THEOREM 1 Suppose that cumulative prospect theory holds. Then the following two statements are equivalent:

1. Strong risk aversion holds.

2. The weighting functions $w^{+}$and $w^{-}$are convex and continuous on the half-open interval $[0,1)$, the utility function is concave on the domain of losses and also on 
the domain of gains. Moreover, the utility function and the weighting functions are differentiable almost everywhere. In particular the left and right derivative of the utility function at any outcome exists, as well as the left and right derivatives of the weighting functions at any $p$ in $(0,1)$. Further, the following relationship is satisfied:

$$
\frac{U^{\prime}\left(0^{-}\right)}{U^{\prime}\left(0^{+}\right)} \geqslant \sup _{p \in(0,1)} \frac{w^{+\prime}\left(p^{+}\right)}{w^{-\prime}\left(p^{-}\right)}
$$

Theorem 1 shows that the utility function does not need to be concave on the entire real line since convexity at the status quo is permitted. In the next section two special cases of CPT are analyzed with respect to this issue and an example with convex utility is derived.

The formula obtained in Theorem 1 can be used to derive a new measure of loss aversion. Since in general $U^{\prime}\left(0^{-}\right) / U^{\prime}\left(0^{+}\right)$can be less than unity also in the case of strict risk aversion some losses, however small they are, may not "loom larger than the corresponding gains", that is, not for all $x>0$ we have $|U(-x)| \geqslant U(x)$. Therefore, loss aversion does only hold if $U^{\prime}\left(0^{-}\right) / U^{\prime}\left(0^{+}\right) \geqslant 1$. Note that an identical definition of loss aversion was already proposed by Bernatzi and Thaler [2] and formalized by Köbberling and Wakker [18]. The latter authors denote the ratio $U^{\prime}\left(0^{-}\right) / U^{\prime}\left(0^{+}\right)$as index of loss aversion (say $\lambda$ ) and employ it in order to compare the degree of loss aversion between different individuals.

If we want to derive a utility function in Theorem 1 which is overall concave then $U^{\prime}\left(0^{-}\right) / U^{\prime}\left(0^{+}\right)$must be larger or equal to 1 . Since the converse relationship holds as well we can formulate the following corollary.

Corollary 2 Assume that CPT holds and that risk aversion is satisfied. Then, the utility function is concave if and only if loss aversion holds. 
Note that in the case of RDU we have $w^{+} \equiv w^{-}$and, therefore, $\sup _{p \in(0,1)} w^{+\prime}\left(p^{+}\right) / w^{-\prime}\left(p^{-}\right) \geqslant$ 1. Consequently, in this case strong risk aversion does always imply loss aversion and a concave utility function on the entire domain.

For CPT, two examples which illustrate the issue of non-concavity of the utility function in a region around the status quo are presented in the next section.

\section{Risk Aversion and Convex Utility}

As already noted in the preceding section, the interest of economists in a particular parametric form for utility has lead to simpler axiomatizations of CPT. The first functional form for utility which we analyze here is linear/exponential utility. Zank [37] provides a characterization of CPT with linear/exponential utility for decision under uncertainty. A function $U: I R \rightarrow I R$ is from the increasing linear/exponential family for gains (losses) if one of the following holds for all $x \geqslant 0(x \leqslant 0)$ :

(i) $U(x)=\alpha x$, with $\alpha>0$,

(ii) $U(x)=\alpha e^{\gamma x}+\tau$, with $\alpha \gamma>0$ and $\tau \in \mathbb{R}$.

Under CPT utility satisfies $U(0)=0$. Therefore, in (i) we dropped the location parameter, and in (ii) the only possibility for the location parameter is $\tau=-\alpha$. In the above definition only the functional form of utility is described. Clearly the parameters $\alpha, \gamma$ can be different for gains (say $\alpha^{+}, \gamma^{+}$) than for losses (say $\alpha^{-}, \gamma^{-}$). The following result holds if we assume CPT with linear/exponential utility in Theorem 1.

COROLlary 3 Suppose that cumulative prospect theory holds with linear/exponential utility. Then the following two statements are equivalent: 
1. Strong risk aversion holds.

2. The weighting functions $w^{+}$and $w^{-}$are convex and continuous on the half-open interval $[0,1)$, the utility function is concave on the domain of losses and concave on the domain of gains. Moreover, the weighting functions are differentiable almost everywhere. In particular the left and right derivative of the utility function at any outcome exists, as well as the left and right derivatives of the weighting functions at any $p$ in $(0,1)$. Further, the following relationship is satisfied:

$$
\lambda:=\left\{\begin{array}{cc}
\alpha^{-} \gamma^{-} / \alpha^{+} \gamma^{+} & \text {if utility is exponential } \\
\alpha^{-} / \alpha^{+} & \text {if utility is linear }
\end{array}\right\} \geqslant \sup _{p \in(0,1)} \frac{w^{+\prime}\left(p^{+}\right)}{w^{-\prime}\left(p^{-}\right)}
$$

where $\lambda$ denotes the index of loss aversion.

If the utility function in the above corollary is exponential for both, gains and losses, then Statement 2, which says that utility is concave on the loss-domain and concave on the gain domain, implies that the involved parameters are all negative. We can use this result to design an example which shows that utility is not necessarily concave on the entire domain. Suppose that for some positive $\alpha$ the utility function is defined as

$$
U(x)=\left\{\begin{aligned}
-\exp (-x)+1, & x \geqslant 0 \\
-\alpha(\exp (-x)-1), & x \leqslant 0
\end{aligned}\right.
$$

and for some positive $\beta$ the weighting functions are defined as

$$
w^{+}(p)=\left\{\begin{array}{cc}
\frac{\exp (p)-1}{4(e-1)}, & p \in[0,1) \\
1, & p=1
\end{array}\right.
$$

and

$$
w^{-}(p)=\left\{\begin{array}{cc}
\beta w^{+}(p), & p \in[0,1) \\
1, & p=1
\end{array}\right.
$$


Obviously, CPT holds and Statement 2 in the above corollary is satisfied if $\alpha \beta \geqslant 1$. Hence, risk aversion holds. However, utility is not concave for $\alpha<1$. Non-concavity of the utility will occur if for example $\alpha=0.9$ and $\beta=2$.

The next corollary is focusing on a result of Wakker and Zank [35], in which utility is a two-sided power function of the following form:

$$
U(x)=\left\{\begin{array}{cc}
\sigma^{+} x^{\alpha}, & \text { with } \sigma^{+}>0, \alpha>0, \text { for all } x \geqslant 0, \\
-\sigma^{-}|x|^{\beta}, & \text { with } \sigma^{-}>0, \beta>0, \text { for all } x \leqslant 0 .
\end{array}\right.
$$

This form for utility under CPT has been proposed by Tversky and Kahneman [33] and is the most used parametric form in empirical and theoretical applications (many references are given in [35]). If we assume CPT with power utility in Theorem 1 then the following result holds.

COROLlary 4 Suppose that cumulative prospect theory holds with power utility. Then the following two statements are equivalent:

\section{Strong risk aversion holds.}

2. The weighting functions $w^{+}$and $w^{-}$are convex and continuous on the half-open interval $[0,1)$, the utility function is linear on the domain of losses and also on the domain of gains. Moreover, the weighting functions are differentiable almost everywhere. In particular the left and right derivative of the utility function at any outcome exists, as well as the left and right derivatives of the weighting functions at any $p$ in $(0,1)$. Further, the following relationship is satisfied:

$$
\lambda:=\frac{\sigma^{-}}{\sigma^{+}} \geqslant \sup _{p \in(0,1)} \frac{w^{+\prime}\left(p^{+}\right)}{w^{-\prime}\left(p^{-}\right)}
$$

where $\lambda$ denotes the index of loss aversion. 
This result shows that risk aversion and convex utility can coexist under CPT. Assume that CPT holds with the following utility function

$$
U(x)=\left\{\begin{array}{l}
\sigma^{+} x, \quad x \geqslant 0, \\
\sigma^{-} x, \quad x \leqslant 0,
\end{array}\right.
$$

and the weighting functions

$$
w^{+}(p)=\left\{\begin{array}{cc}
p / 4, & p \in[0,1), \\
1, & p=1
\end{array}\right.
$$

and

$$
w^{-}(p)=\left\{\begin{array}{cc}
p / 2, & p \in[0,1), \\
1, & p=1 .
\end{array}\right.
$$

Then for $\sigma^{+}=1, \sigma^{-}=0.9$ the conditions in Statement 2 of the above corollary are satisfied, and obviously utility is convex, due to the fact that utility is less steep for losses than for gains (i.e. $1=\sigma^{+}>\sigma^{-}=0.9$ ).

The reason why in this example we have loss seeking is that the weighting functions are allowed to be discontinuous at 1 . If we require continuity of the weighting functions on $[0,1]$, as is often done in the literature, loss-seeking behavior cannot occur. In that case continuity and convexity of the weighting functions imply that $\sup _{p \in(0,1)}\left[w^{+\prime}\left(p^{+}\right) / w^{-\prime}\left(p^{-}\right)\right]$ will be at least 1 , and utility must be concave on the entire domain. The proof is simple. If $w^{-}$is above $w^{+}$then for $p$ close to 1 we have $w^{-1}\left(p^{-}\right) \leqslant w^{+\prime}\left(p^{+}\right)$. Similarly, if $w^{-}$is below $w^{+}$then for $p$ close to 0 we have $w^{-\prime}\left(p^{-}\right) \leqslant w^{+\prime}\left(p^{+}\right)$. If neither of the previous cases holds, then the two weighting functions must intersect. In that case there exists an interval in $[0,1]$ where either $w^{-}$is above $w^{+}$or $w^{-}$is below $w^{+}$and where $w^{-}$is below $w^{+}$at the boundary of the interval. Using similar arguments as above, we can conclude the existence of some $p \in(0,1)$ with $w^{-1}\left(p^{-}\right) \leqslant w^{+\prime}\left(p^{+}\right)$. 
This analysis is independent of the chosen utility function, and therefore it holds in general. This shows that, while under RDU assuming a continuous or discontinuous weighting function is irrelevant for the shape of the utility function, here the assumption of continuous weighting functions is crucial and forces utility to be concave. We get the following result.

LEmma 5 Suppose that CPT holds and that strong risk aversion is satisfied. Further, assume that the weighting functions are continuous on $[0,1]$. Then, loss seeking behavior is excluded, i.e. the utility function is concave.

\section{Appendix. Proofs}

Proof of Theorem 1: Let us first assume Statement 1 and derive Statement 2. Suppose strong risk aversion holds and as well CPT. In what follows we prove that if the outcomes are gains or zero then utility must be concave and the weighting function $w^{+}$ convex. Then a similar result is derived for the case of losses (or zero): again utility is concave and the weighting function $w^{-}$is convex. In both cases we cannot rely on results from the literature as our assumptions are weaker: we do not assume differentiability of the utility function, neither do we assume continuity of the weighting functions. We will show that the additional assumptions are not necessary.(It has been shown in Lemma 5 that such additional assumptions are rather restrictive under CPT.)

The final step in the derivation of Statement 2 is to consider the mixed outcome case.

\section{Step 1: Outcomes are gains or they are the status quo.}

First we consider the lottery $P:=\left(p_{1}, x_{1} ; \ldots ; p_{i}, x_{i} ; p_{i+1}, x_{i+1} ; \ldots ; p_{n}, x_{n}\right)$. Strong risk 
aversion implies that

$$
\left(p_{1}, x_{1} ; \ldots ; p_{i}, x_{i}-\frac{\delta}{p_{i}} ; p_{i+1}, x_{i+1}+\frac{\delta}{p_{i+1}} ; \ldots ; p_{n}, x_{n}\right) \succcurlyeq P
$$

whenever $\delta \in\left[0,\left(x_{i}-x_{i+1}\right) / 2\right]$. After elimination of common terms, substitution of CPT gives

$$
\pi_{i}\left[U\left(x_{i}\right)-U\left(x_{i}-\frac{\delta}{p_{i}}\right)\right] \leqslant \pi_{i+1}\left[U\left(x_{i+1}+\frac{\delta}{p_{i+1}}\right)-U\left(x_{i+1}\right)\right]
$$

or equivalently

$$
\frac{p_{i+1} \pi_{i}}{p_{i} \pi_{i+1}} \leqslant \frac{\left[U\left(x_{i+1}+\delta / p_{i+1}\right)-U\left(x_{i+1}\right)\right]}{\delta / p_{i+1}} \frac{\delta / p_{i}}{\left[U\left(x_{i}\right)-U\left(x_{i}-\delta / p_{i}\right)\right]}
$$

Now we use the fact that the utility function is continuous and strictly increasing, hence differentiable almost everywhere. Then we can choose $x_{i}, x_{i+1}$ such that derivative at those values is well defined. Then, for $\delta \rightarrow 0$ it must hold that

$$
\frac{p_{i+1} \pi_{i}}{p_{i} \pi_{i+1}} \leqslant \frac{U^{\prime}\left(x_{i+1}\right)}{U^{\prime}\left(x_{i}\right)}
$$

and by letting $x_{i} \rightarrow x_{i+1}$ in the limit, we find

$$
\frac{p_{i+1} \pi_{i}}{p_{i} \pi_{i+1}} \leqslant 1
$$

or

$$
\frac{\pi_{i}}{p_{i}} \leqslant \frac{\pi_{i+1}}{p_{i+1}}
$$

This shows that for any selection of probabilities we have

$$
\frac{w^{+}\left(\sum_{j=1}^{i} p_{j}\right)-w^{+}\left(\sum_{j=1}^{i-1} p_{j}\right)}{p_{i}} \leqslant \frac{w^{+}\left(\sum_{j=1}^{i+1} p_{j}\right)-w^{+}\left(\sum_{j=1}^{i} p_{j}\right)}{p_{i+1}}
$$

or equivalently that the weighting function $w^{+}$is convex.

It can now be shown that the weighting function $w^{+}$, which is strictly increasing satisfying $w^{+}(0)=0$ and $w^{+}(1)=1$, is continuous on $[0,1)$. Convexity and monotonicity 
are sufficient for the derivation of this property. Now we use the fact that a monotonic continuous function is differentiable almost everywhere. In addition the weighting function $w^{+}$is convex, and this means that its left and right derivative at each point in $(0,1)$ exists as well as the right derivative at 0 .

For this case it remains to show that the utility function is concave. Strong risk aversion implies that

$$
\left(1 / 2, x_{1}-\delta ; 1 / 2, x_{2}+\delta\right) \succcurlyeq\left(1 / 2, x_{1} ; 1 / 2, x_{2}\right)
$$

whenever $\delta \in\left[0,\left(x_{1}-x_{2}\right) / 2\right]$. After elimination of common terms, substitution of CPT gives

$$
\left[w^{+}(1 / 2)\right]\left[U\left(x_{1}\right)-U\left(x_{1}-\delta\right)\right] \leqslant\left[1-w^{+}(1 / 2)\right]\left[U\left(x_{2}+\delta\right)-U\left(x_{2}\right)\right]
$$

and using the convexity of $w^{+}$and the continuity at $1 / 2$ we find

$$
1 \leqslant \frac{\left[U\left(x_{2}+\delta\right)-U\left(x_{2}\right)\right]}{\left[U\left(x_{1}\right)-U\left(x_{1}-\delta\right)\right]}
$$

With $\delta=1 / 2\left(x_{1}-x_{2}\right)$ we derive

$$
\frac{U\left(x_{1}\right)+U\left(x_{2}\right)}{2} \leqslant U\left(\frac{\left(x_{1}+x_{2}\right)}{2}\right),
$$

which implies concavity of the utility function, as $x_{i}, x_{i+1}$ were arbitrary (gains).

In order to show that the left and right derivative of $U$ exists at any gain outcome, note that a similar argument as in the case of the weighting function $w^{+}$applies here. However, because the domain of the utility function is unbounded from above, we conclude that the left and right derivatives of the utility function at each point $x>0$ exist and in particular the right derivative at the status quo is well defined.

\section{Step 2: Outcomes are losses or they are the status quo.}


The proof is similar to the one in the previous case. Therefore, we can conclude that the utility function is concave for losses and that the weighting function $w^{-}$is convex. Moreover, continuity of $w^{-}$on $[0,1)$ is satisfied and the left and right derivative of $w^{-}$ exists for each point in $(0,1)$, in particular the right derivative of $w^{-}$at 0 is well defined. Similarly to the previous case we can conclude that the utility function $U$ is differentiable almost everywhere, and that the left and right derivative exists at any $x<0$. In addition the left derivative of $U$ at 0 is well defined, and in general it may not agree with the right derivative established in step 1 of this proof.

\section{Step 2: Outcomes are gains or losses.}

This step will focus entirely on the derivation of the inequality in Statement 2 of the theorem. Suppose we have a lottery $P=\left(p_{1}, x_{1} ; \ldots ; p_{k}, x_{k} ; p_{k+1}, x_{k+1} ; \ldots ; p_{n}, x_{n}\right)$, where $x_{k}>0>x_{k+1}$. Then strong risk aversion implies

$$
\left(p_{1}, x_{1} ; \ldots ; p_{k}, x_{k}-\frac{\delta}{p_{k}} ; p_{k+1}, x_{k+1}+\frac{\delta}{p_{k+1}} ; \ldots ; p_{n}, x_{n}\right) \succcurlyeq P .
$$

Let now $\delta$ be small enough such that $x_{k}-\delta>0>x_{k+1}+\delta$. Substitution of CPT gives

$$
\pi_{k}\left[U\left(x_{k}\right)-U\left(x_{k}-\frac{\delta}{p_{k}}\right)\right] \leqslant \pi_{k+1}\left[U\left(x_{k+1}+\frac{\delta}{p_{k+1}}\right)-U\left(x_{k+1}\right)\right]
$$

Therefore, for any $x_{k}>0>x_{k+1}$ and any probabilities $p_{k}, p_{k+1}$ the following must be satisfied:

$$
\frac{\left[U\left(x_{k}\right)-U\left(x_{k}-\frac{\delta}{p_{k}}\right)\right]}{\left[U\left(x_{k+1}+\frac{\delta}{p_{k+1}}\right)-U\left(x_{k+1}\right)\right]} \leqslant \frac{\left[w^{-}\left(\sum_{j=1}^{k+1} p_{j}\right)-w^{-}\left(\sum_{j=1}^{k} p_{j}\right)\right]}{\left[w^{+}\left(\sum_{j=1}^{k} p_{j}\right)-w^{+}\left(\sum_{j=1}^{k-1} p_{j}\right)\right]} .
$$

We can write this inequality as

$$
\frac{\left[U\left(x_{k}\right)-U\left(x_{k}-\frac{\delta}{p_{k}}\right)\right] / \frac{\delta}{p_{k}}}{\left[U\left(x_{k+1}+\frac{\delta}{p_{k+1}}\right)-U\left(x_{k+1}\right)\right] / \frac{\delta}{p_{k+1}}} \leqslant \frac{\left[w^{-}\left(\sum_{j=1}^{k+1} p_{j}\right)-w^{-}\left(\sum_{j=1}^{k} p_{j}\right)\right] / p_{k+1}}{\left[w^{+}\left(\sum_{j=1}^{k} p_{j}\right)-w^{+}\left(\sum_{j=1}^{k-1} p_{j}\right)\right] / p_{k}}
$$


This inequality needs to be satisfied for all $x_{k}>0>x_{k+1}$, all probabilities $p_{1}, \ldots, p_{n}$ and any appropriate $\delta$. Hence,

$$
\sup _{\substack{x_{k}>0>x_{k+1} \\ \delta>0}} \frac{\left[U\left(x_{k}\right)-U\left(x_{k}-\frac{\delta}{p_{k}}\right)\right] / \frac{\delta}{p_{k}}}{\left[U\left(x_{k+1}+\frac{\delta}{p_{k+1}}\right)-U\left(x_{k+1}\right)\right] / \frac{\delta}{p_{k+1}}} \leqslant \inf _{p_{1}, \ldots, p_{n}} \frac{\left[w^{-}\left(\sum_{j=1}^{k+1} p_{j}\right)-w^{-}\left(\sum_{j=1}^{k} p_{j}\right)\right] / p_{k+1}}{\left[w^{+}\left(\sum_{j=1}^{k} p_{j}\right)-w^{+}\left(\sum_{j=1}^{k-1} p_{j}\right)\right] / p_{k}}
$$

must hold. We now use the concavity of the utility function and the convexity of the weighting functions and derive

$$
\sup _{x_{k}>0>x_{k+1}} \frac{U^{\prime}\left(x_{k}^{+}\right)}{U^{\prime}\left(x_{k+1}^{-}\right)} \leqslant \inf _{p \in(0,1)} \frac{w^{-\prime}\left(p^{-}\right)}{w^{+\prime}\left(p^{+}\right)}
$$

or further using concavity of the utility function on the gain domain and on the loss domain:

$$
\frac{U^{\prime}\left(0^{+}\right)}{U^{\prime}\left(0^{-}\right)} \leqslant \inf _{p \in(0,1)} \frac{w^{-\prime}\left(p^{-}\right)}{w^{+\prime}\left(p^{+}\right)}
$$

This inequality is equivalent to

$$
\frac{U^{\prime}\left(0^{-}\right)}{U^{\prime}\left(0^{+}\right)} \leqslant \sup _{p \in(0,1)} \frac{w^{+\prime}\left(p^{+}\right)}{w^{-\prime}\left(p^{-}\right)}
$$

which concludes the proof of Statement 2.

Let us now assume that Statement 2 holds. The proof of Statement 1 follows from the fact that under CPT strong risk aversion is equivalent to

$$
\frac{p_{j} \pi_{i}}{p_{i} \pi_{j}} \leqslant \frac{\left[U\left(x_{j}+\delta / p_{j}\right)-U\left(x_{j}\right)\right]}{\delta / p_{j}} \frac{\delta / p_{i}}{\left[U\left(x_{i}\right)-U\left(x_{i}-\delta / p_{i}\right)\right]},
$$

for any $x_{i}>x_{j}$ and probabilities $p_{i}+p_{j} \leqslant 1$, and appropriate $\delta>0$. For each of the three cases $\left(x_{i}>x_{j}>0,0>x_{i}>x_{j}, x_{i}>0>x_{j}\right)$ this inequality follows from continuity of the different functions and the convexity of the weighting functions together with the concavity of $U$ for gains and for losses. For the derivation of the case $x_{i}>0>x_{j}$ the inequality in statement 2 of the theorem is needed. This concludes the proof of Theorem 1. 
Proof of Corollary 2: The proof follows immediately from the new definition of loss aversion: $\lambda=U^{\prime}\left(0^{-}\right) / U^{\prime}\left(0^{+}\right)$.

Proof of Corollary 3: The proof follows from substitution of the particular form for the utility function in Theorem 1.

Proof of Corollary 4: The proof follows from substitution of the particular form for the utility function in Theorem 1.

\section{Acknowledgments:}

This paper was written during the first author's visit at the School of Economic Studies, University of Manchester. Financial support for this visit under The Manchester School Visiting Research Fellow Scheme is gratefully acknowledged. We are indebted to Mark J. Machina and Michèle Cohen for helpful comments and suggestions on an earlier version of this paper.

\section{References}

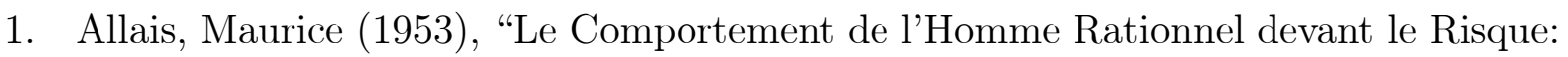
Critiquedes Postulats et AxiomesđelPEcole Américaine," Econometrica 21, 503-546.

2. $\square$ Benartzi, Shlomo\& RichardH.Thaler(1995), "Myopic Loss Aversionand the Equity Premium Puzzle," Quarterly Journal of Economics 110, 73-92.

3. $\square$ Chateauneuf, $\square$ Alain $\square$ \& $\square$ Michèle $\square$ Cohen $\square(1994)$, $\square$ "Risk $\square$ Seeking $\square$ with $\square$ Diminishing Marginal Utility in a Non-Expected Utility Model," Journal of Risk and Uncertainty 9, 77-91. 


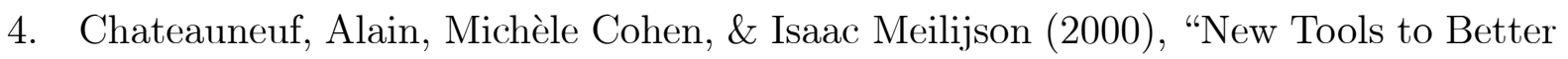
Model Behavior Under Risk and Uncertainty: An Overview," mimeo, University of Paris I.

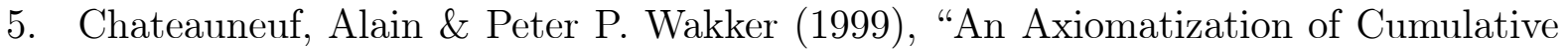
Prospect Theory for Decision under Risk," Journal of Risk and Uncertainty 18, 137145.

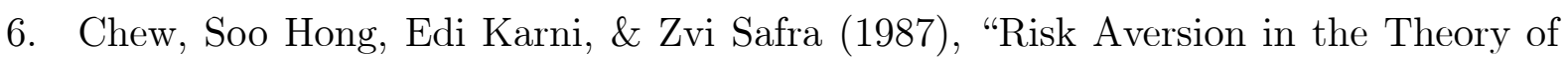
Expected Utility with Rank Dependent Probabilities," Journal of Economic Theory $42,370-381$.

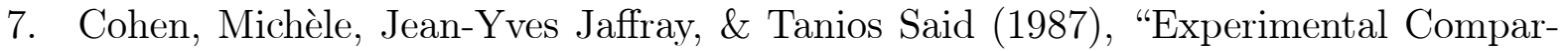
isons of Individual Behavior under Risk and under Uncertainty for Gains and for Losses," Organizational Behavior and Human Decision Processes 39, 1-22.

8. $\square$ Dunn, LuciaF. (1996), $\longleftarrow$ Loss Aversionand Adaptation in the Labour Market:Empirical Indifference Functions and Labour Supply," Review of Economics and Statistics $78,441-450$.

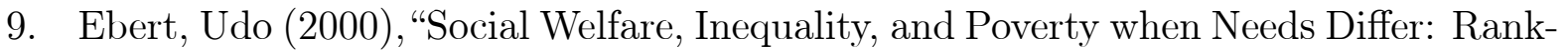
Dependent Evaluation Functions," Dept. of Economics, University of Oldenburg, Germany.

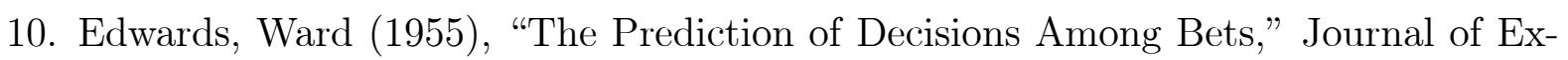
perimental Psychology 50, 201-214.

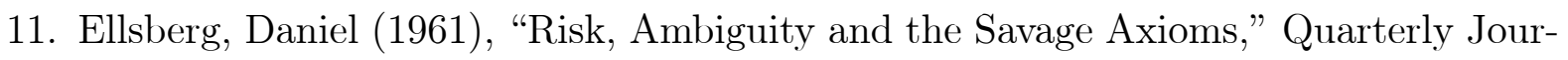
nal of Economics 75, 643-669. 


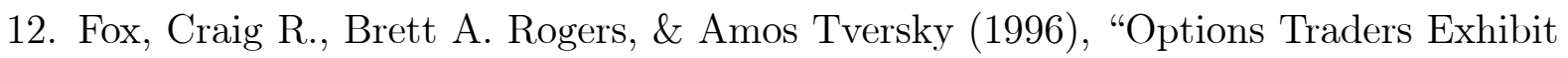
Subadditive Decision Weights," Journal of Risk and Uncertainty 13, 5-17.

13. $\square$ Friedman, $\square$ Milton $\& \square$ Leonard $\square$ J. $\square$ Savage $\square(1948), \square$ "The $\square$ Utility $\square$ Analysis $\square$ of $\square$ Choices Involving Risk," Journal of Political Economy 56, 279-304.

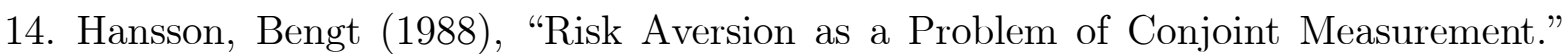
In Peter Gärdenfors \& Nils-Eric Sahlin (Eds.), Decision, Probability, and Utility; Selected Readings, 136- 158, Cambridge University Press, Cambridge.

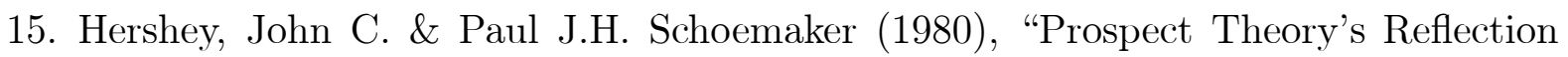
Hypothesis: A Critical Examination," Organizational Behavior and Human Performance 25, 395-418.

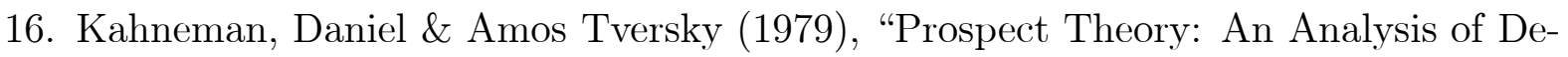
cision under Risk," Econometrica 47, 263-291.

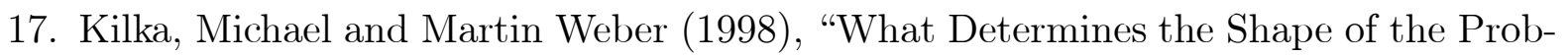
ability Weighting Function under Uncertainty," University of Mannheim, Mannheim, Germany.

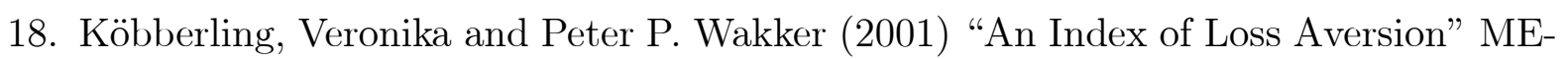
TEOR, Maastricht University, The Netherlands.

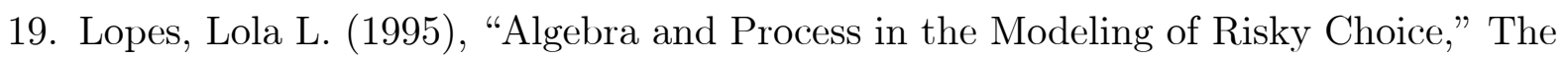
Psychology of Learning and Motivation 32, 177-220. 


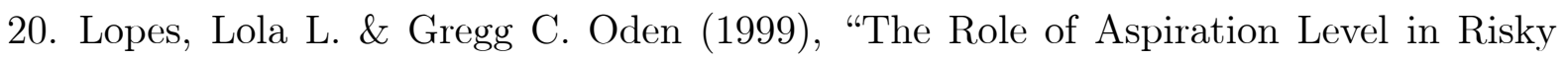
Choice: A Comparison of Cumulative Prospect Theory and SP/A Theory," Journal of Mathematical Psychology 43, 286-313.

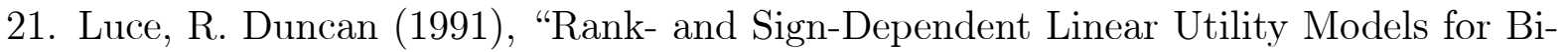
nary Gambles," Journal of Economic Theory 53, 75-100.

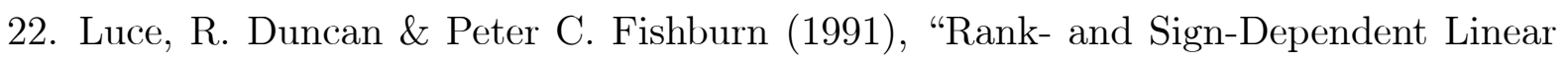
Utility Models for Finite First-Order Gambles," Journal of Risk and Uncertainty 4, 29-59.

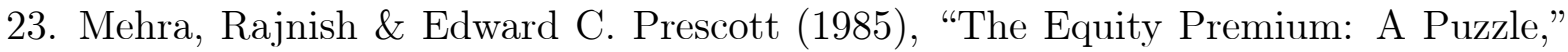
Journal of Monetary Economics 15, 145-162.

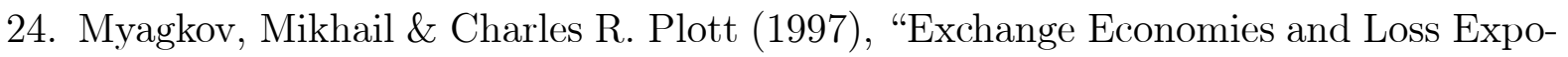
sure: Experiments Exploring Prospect Theory and Competitive Equilibria in Market Environments," American Economic Review 87, 801-828.

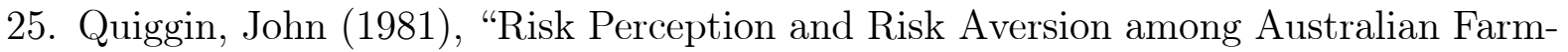
ers," Australian Journal of Agricultural Economics 25, 160-169.

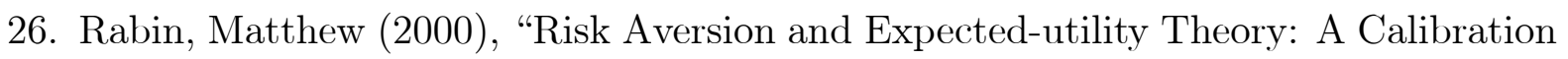
Theorem," Econometrica 68, 1281-1292.

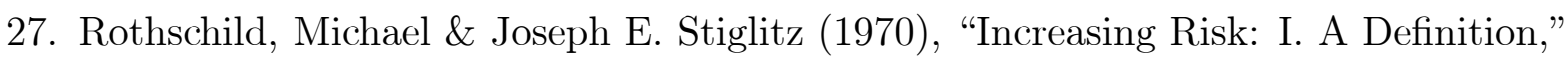
Journal of Economic Theory 2, 225-243.

28. Samuelson, William F. \& Richard J.ZZeckhauser (1988), ॠStatus QuoBias in Decision Making," Journal of Risk and Uncertainty 1, 7-59. 
29. $₫$ Schmidt, $\square$ Ulrich $\square(2001), \square$ Reference-Dependence $\_$in $\square$ Cumulative $\triangle$ Prospect $\square$ Theory," mimeo.

30. $\square$ Schmidt, $\square$ Ulrich $\& \square$ Horst ZZank $\square$ 2001), $\square$ "An $\square$ Axiomatization $\curvearrowleft$ f $\square$ Linear $\square$ Cumulative Prospect Theory with Applications to Portfolio Selection and Insurance Demand", mimeo.

31. Schneider, SandraL.\&LolaL.Lopes (1986), ॠReflection in Preferences under Risk: Who and When May Suggest Why," Journal of Experimental Psychology: Human Perception and Performance 12, 535-548.

32. Thaler, Richard H. (1980), ॠTowardaPositive Theory of Consumer Choice," Journal of Economic Behavior and Organization 1, 39-60.

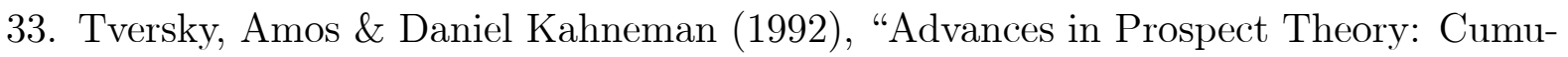
lative Representation of Uncertainty," Journal of Risk and Uncertainty 5, 297-323.

34. $\square$ Wakker, $\square$ Peter $\square$ P. $\square \& \square$ Amos $\square$ Tversky $\square(1993), \square$ “An $\square$ Axiomatization $\square$ of $\square$ Cumulative Prospect Theory," Journal of Risk and Uncertainty 7, 147-176.

35. $\square$ Wakker, Peter P. \& Horst Zank (2001), ॠA Simple Preference-Foundation of Cumulative Prospect Theory with Power Utility," European Economic Review, forthcoming.

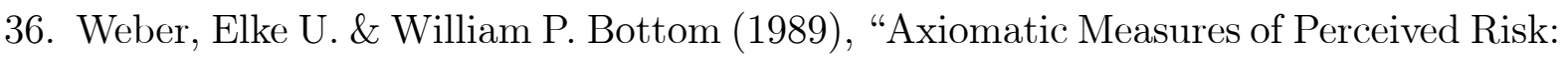
Some Tests and Extensions," Journal of Behavioral Decision Making 2, 113-131.

37. Zank, Horst (2001), $\nleftarrow$ Cumulative Prospect Theory for Parametric and Multiattribute Utilities," Mathematics of Operations Research 26, No. 1, 67-81. 